\title{
Three Dimensional Data Capture in Indoor Environments for Autonomous Navigation
}

\section{J.M. Evans \\ T. Chang \\ T.H. Hong \\ R. Bostelman \\ W.R. Bunch}

U. S. DEPARTMENT OF COMMERCE

Technology Administration

National Institute of Standards and Technology

Intelligent Systems Division

Gaithersburg, MD 20899-8230

\section{$Q C$}

100

.456

*6912

2002

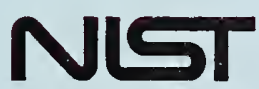

National Instifute of Standards and Technology

Technology Administration

U.S. Department of Commerce 



\section{Three Dimensional Data Capture in Indoor Environments for Autonomous Navigation}

J.M. Evans

T. Chang

T.H. Hong

R. Bostelman

W.R. Bunch

U. S. DEPARTMENT OF COMMERCE

Technology Administration

National Institute of Standards

and Technology

Intelligent Systems Division

Gaithersburg, MD 20899-8230

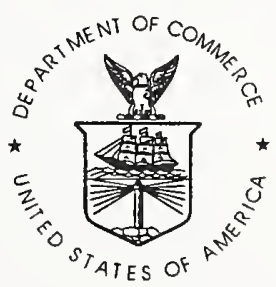

U.S. DEPARTMENT OF COMMERCE Donald L. Evans, Secretary

TECHNOLOGY ADMINISTRATION

Phillip J. Bond, Under Secretary for Technology

NATIONAL INSTITUTE OF STANDARDS

AND TECHNOLOGY

Arden L. Bement, Jr., Director 



\section{Three Dimensional Data Capture in Indoor Environments for Autonomous Navigation \\ NIST Internal Report \#6912 \\ Evans, J.M., Chang, T., Hong, T.H., Bostelman, R. and Bunch, W.R. Jevans@nist.gov, tchang@nist.gov, thong@nist.gov, bostel@nist.gov, bunch@nist.gov \\ Intelligent Systems Division \\ National Institute of Standards and Technology \\ 100 Bureau Drive, Mail Stop 8230 \\ Gaithersburg, Maryland, 20899}

\begin{abstract}
An innovative use of a low-cost laser range scanner captures three-dimensional features of indoor environments in a manner suitable for mobile robot navigation that is superior to earlier approaches. This three dimensional mapping solves many of the known problems commonly encountered in early commercial service robot products. Examples include overhangs such as tables and water fountains, obstacles on the floor below the height of the scanner, carts with open sides and such items as $I V$ poles in the hospitals. This paper describes the mapping procedure and presents early test results.
\end{abstract}

\section{Background}

Research in autonomous mobile robots started in the 1960's [1] and has been widespread since the early 1980's. Despite thousands of man-years of work and thousands of publications covering this work, commercial applications have generally failed. There are two reasons for this: first, much of the research work has focused on mapping, path planning algorithms and behavior generation while the limiting real world problems were with sensing and perception. Second, economic justification and marketplace success require a combination of low prices with high functionality and high reliability, a combination which has not been achieved.

Most of the work in the 1980 s and well into the 1990 's employed ultrasonic range sensors for indoor navigation and machine vision for outdoor navigation [2-7]. The introduction of ladar (laser radar) sensors in the early 1990's has resulted in a new paradigm for mobile robot navigation [8]. The
SICK Optic ${ }^{1}$ laser scanner in particular has become common in research laboratories around the world. This instrument can be configured to scan a $180^{\circ}$ radial field of view in a plane at resolutions of $0.5^{\circ}$ or $1^{\circ}$ or a $100^{\circ}$ field of view at a resolution of $0.25^{\circ}$, $0.5^{\circ}$, or $1^{\circ}$. The range can be set to $8.1 \mathrm{~m}, 81 \mathrm{~m}$ or $150 \mathrm{~m}$ and scans are generated at approximately 77 $\mathrm{Hz}[9]$.

\section{Typical Use of Scanning Ladar}

The typical use of a scanning ladar sensor is with the scanning plane parallel to the floor. This provides a good, high resolution profile map of walls and obstacles [10] that is satisfactory for a two dimensional world. That is, if every object in the environment has a cylindrical form, such that all surfaces are vertical and a single horizontal plane cut through the environment adequately captures the extent of all objects, then a two dimensional projection of that data provides a suitable map for path planning by a mobile robot. A typical map generated by the scanning ladar is shown in Figure 1. This is a view of a hallway with an open double door ahead of the sensor.

The real world presents many exceptions that are problematic for mobile robots. Overhangs such as tables and water fountains, obstacles on the floor below the height of the scanner, carts with open sides and items such as IV poles in hospitals (which

\footnotetext{
${ }^{1}$ Certain commercial companies and their products are identified in this paper in order to describe the experimental work adequately. Such identification is not intended to imply any judgement by the National Institute of Standards and Technology concerning the comparative quality of these products, nor is it intended to imply that the products identified are necessarily the best available for the purpose.
} 
have a slender pole mounted an a large castered base) are examples of such problems. [11]

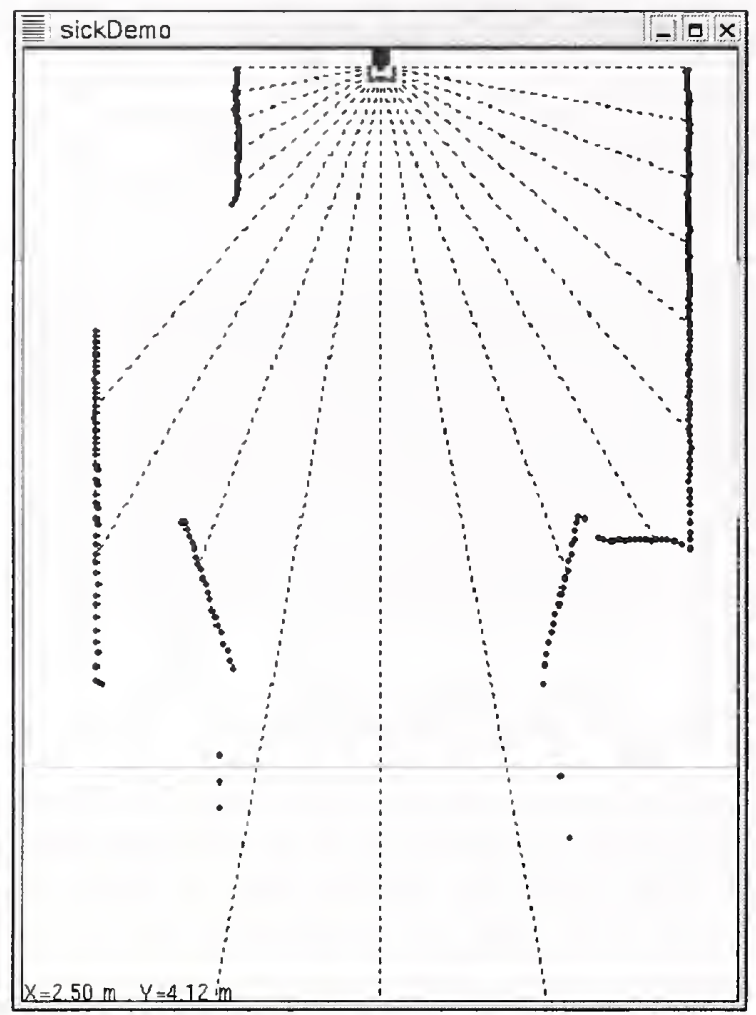

Figure 1 Laser scanner range measurements in a plane

\section{Using a Two Dimensional Scanner for Capturing Three Dimensional Data}

Thrun, Burgard and Fox have demonstrated the use of two laser scanners to generate three dimensional maps of an indoor environment, with one scanner mounted to view a horizontal plane and a second scanner mounted to view a vertical plane. [11]

A new use of a laser scanner to capture three dimensional features that uses only a single scanner has been explored at the National Institute of Standards and Technology (NIST). Mounting the scanner on a mobile robot base with the scanning plane angled down toward the direction of motion provides a "cowcatcher" that sweeps out a volume of space in front of the robot as it moves forward. Accumulating the data in a two and a half or three dimensional map provides a comprehensive model of the world that overcomes many of the problems encountered in the field by early mobile robot pioneers.

The mapping procedure consists of a coordinate transformation, a fusion and a scrolling procedure. The coordinate transformation procedure involves sensor-to-vehicle and vehicle-to-world transformations. Figure 2 shows the geometric relationship between the sensor and the vehicle coordinate systems. The sensor coordinate frame is defined to have the xy plane as the scanning plane. The sensor provides range at a given angle, which is transformed into $x$ and $y$ values. Given the sensor tilt angle $\theta$ (defined as a positive number), and the height $H$, the sensor-to-vehicle 3D homogeneous transformation is the combination of a rotation along the $\mathrm{x}$-axis and a translation along the $\mathrm{z}$-axis:

$\begin{array}{cccc}1 & 0 & 0 & 0 \\ 0 & \cos (-\theta) & -\sin (-\theta) & 0 \\ 0 & \sin (-\theta) & \cos (-\theta) & H \\ 0 & 0 & 0 & 1\end{array}$

Multiplying this transformation matrix by the position vector $[\mathrm{x}, \mathrm{y}, \mathrm{z}, 1]$ yields the point positions in vehicle coordinates. Note that $x$ values are not changed by this transformation but $\mathrm{y}$ and $\mathrm{z}$ values are. 


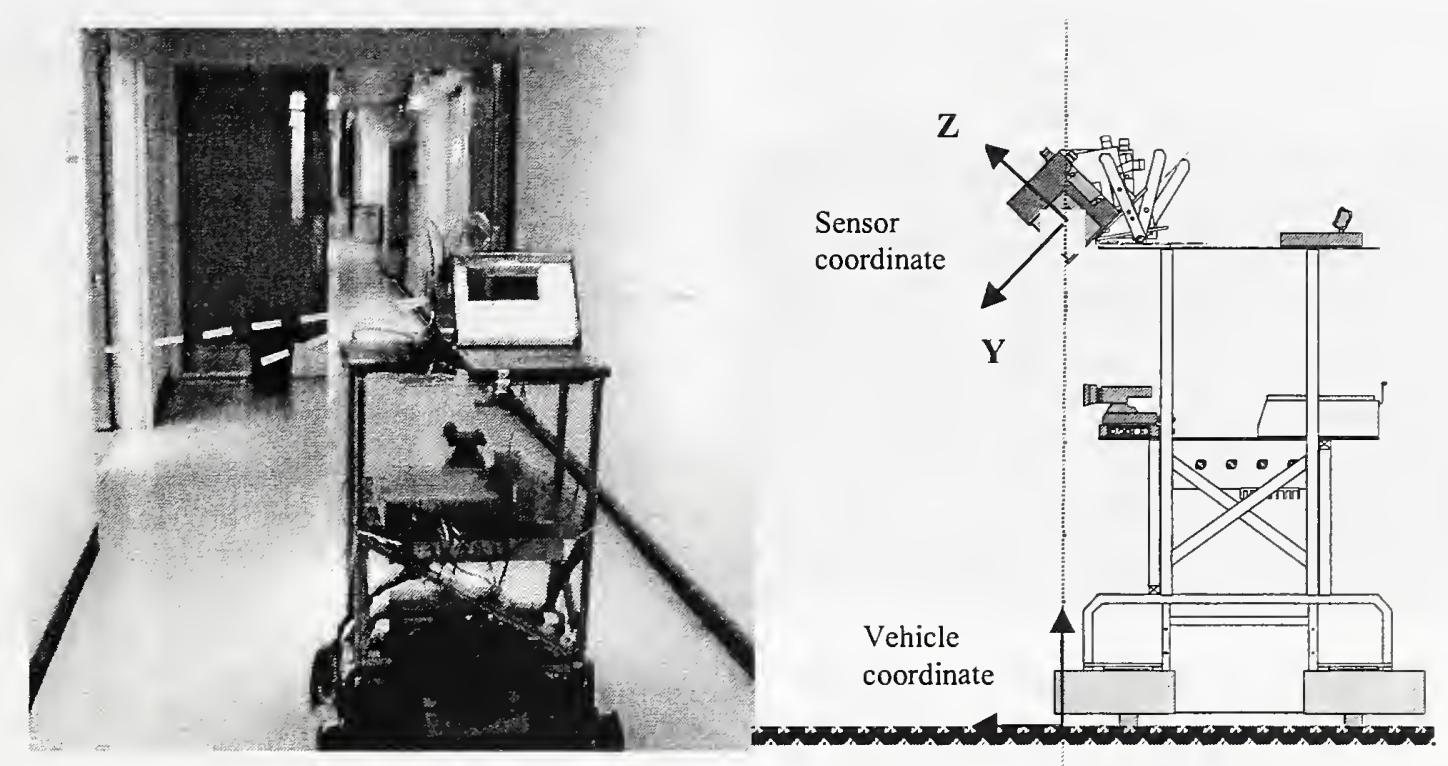

Figure 2. Sensor and vehicle coordinate systems

The sensed data in vehicle coordinates are directly useful for obstacle avoidance if obstacle avoidance algorithms are formulated in a vehicle coordinate frame, as for example the polar coordinate scheme of Borenstein [12]. For mapping or obstacle avoidance using algorithms formulated in a world coordinate frame, a subsequent vehicle-to-world transformation must be made.

NIST work in autonomous mobility has focused on research supporting Demo III, [13] the Army's Experimental Unmanned Ground Vehicle (XUV) development program. Demo III is targeting the development of an autonomous robot vehicle for reconnaissance, surveillance, and target acquisition. Off-road navigation for Demo III is more difficult than planar navigation in an indoor environment, with more potential navigation problems in the terrain, but the basic approach can be used for indoor navigation as well. Demo III uses a multiresolutional $21 / 2 \mathrm{D}$ world model, in which elevation is a composite cost function of terrain elevation, soil properties, obstacles, slope, observability by enemy forces, and other factors. A 2 1/2 D model will be much simpler for indoor navigation with elevation from the floor plane being the most significant state variable.

The vehicle-to-world transformation transforms data from the vehicle coordinate to a world coordinate system. After the transformations, sensed data are updated and fused into the world model [14], which represents the three-dimensional environment in a way suitable for path planning.

The world model contains layers of maps. Examples include the elevation map, the obstacle map and the hallway map. For example, each cell in the elevation map contains basic geometric information such as the average elevation height, the $\min$ and $\max$ elevation values and a confidence measure reflecting the "goodness" of the elevation data. The confidence measure is helpful in dealing with sensor noise as well as moving obstacles.

All of the sensor data, from laser scanners, sonar, vision sensors and bumpers are added into the maps. A priori data, a map of a building layout for example, can be similarly added into the world model maps. The maps are then fused to create a single "best" representation of the world that is used for path planning. For Demo III path planning is done using cost-based search [15]. Simpler approaches can be used for indoor navigation. For example, all of the data can be directly entered into a single local map, with appropriate weights, and path planning can be reduced to figuring deviations from a straight line down hallway necessary to avoid obstacles [16]. 
The map fusion algorithm used at NIST is based on the concept of confidence-based mapping described in Oskard [17]. In this algorithm, confidence measures increase or decrease linearly as the model receives updated information from the sensors. When a map cell receives a vote for a class such as an obstacle, an elevation measurement, a terrain classification, etc., the cell's confidence in that class is incremented by a predefined constant based on the reliability of the sensor. The elevation confidence of each grid cell is updated every sensor cycle, e.g., 75 $\mathrm{Hz}$ for SICK data. The new elevation is updated by the weighted average of the current sensed elevation value and the accumulated elevation value.

A map cell is classified into an object type. Examples of object types are walls, tables, water fountains, etc. The confidence of these classes is updated based on the results of SICK classification algorithms. Confidence values increase by a factor determined by sensor characteristics, which are learned off-line by analyzing and testing data collected for this purpose. Confidence values are used as a cost factor in determining the traversability of a grid cell. The traversability was used in the DEMOIII path planner [15].

A scrolling local map that incorporates new sensor data while keeping the vehicle centered on the map is used. This scrolling approach has the advantage of minimizing cell relocation. No copying of data is done, only updating. When the vehicle moves out of the center cell, the scrolling function is enabled. The scrolling function includes re-centering the map and reinitializing the map borders. Because the map is vehicle-centered, only the borders of the map contain new regions that must be initialized [13].

\section{Experimental Data}

Figure 3 shows a single scan and a maximum elevation map as the robot moves about $1 \mathrm{~m}$ through a hallway at NIST. The virtual robot shown in the map is roughly $40 \mathrm{~cm} \mathrm{x} 40 \mathrm{~cm} \times 20 \mathrm{~cm}$. In the scan plot, the laser scanner is mounted $1.1 \mathrm{~m}$ above the floor and tilted down $12^{\circ}$. This maximum elevation map can be thought of in terms of dropping a curtain wall from every measured point to the floor, effectively creating a complete wall from the set of discrete measured points on that wall. This representation creates a $21 / 2 \mathrm{D}$ map in the plane of the floor that includes the projection of all three dimensional objects that might be obstacles for the mobile robot. Light gray is sensed data, dark is unknown space, vehicle icon is centered. Note that the sensor cannot see the floor directly in front of the robot when it is initialized. It only provides a complete volume sweep as the robot moves down the hallway.
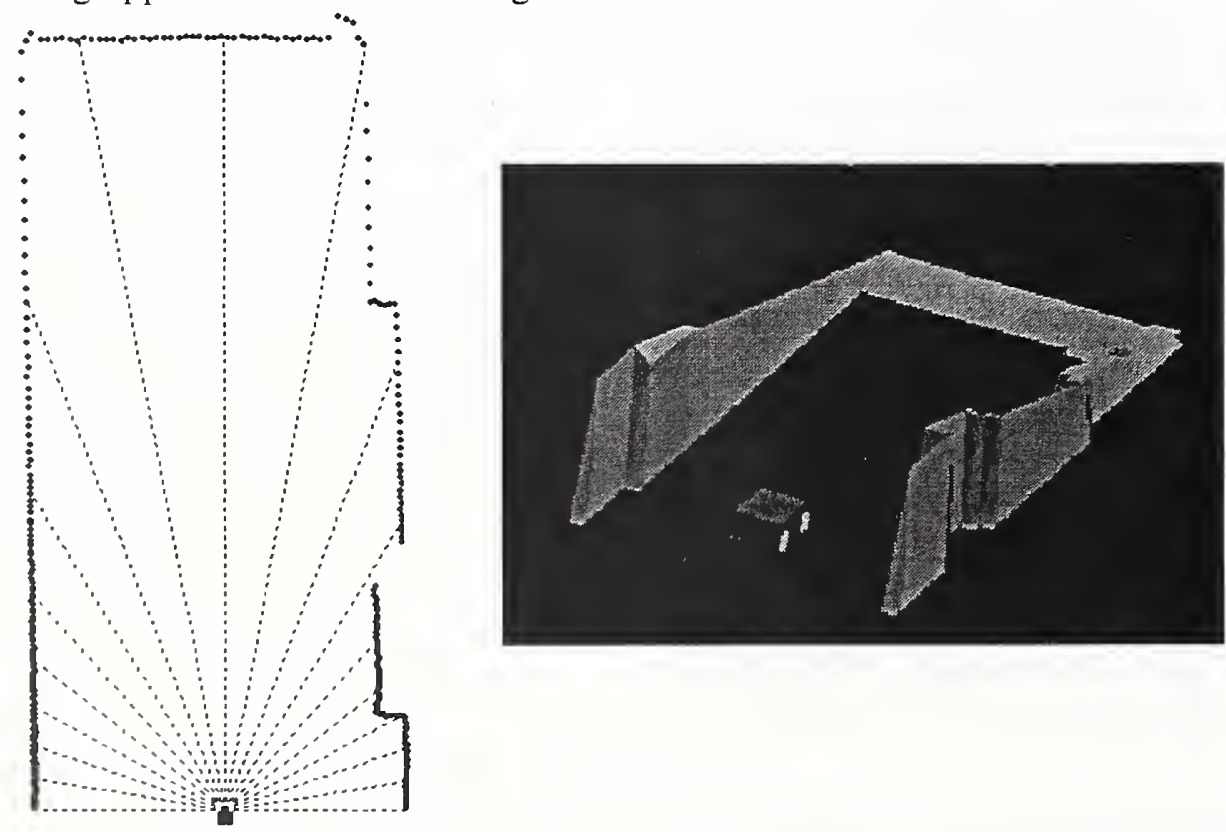

Figure 3. Beginning hallway scan and elevation map 


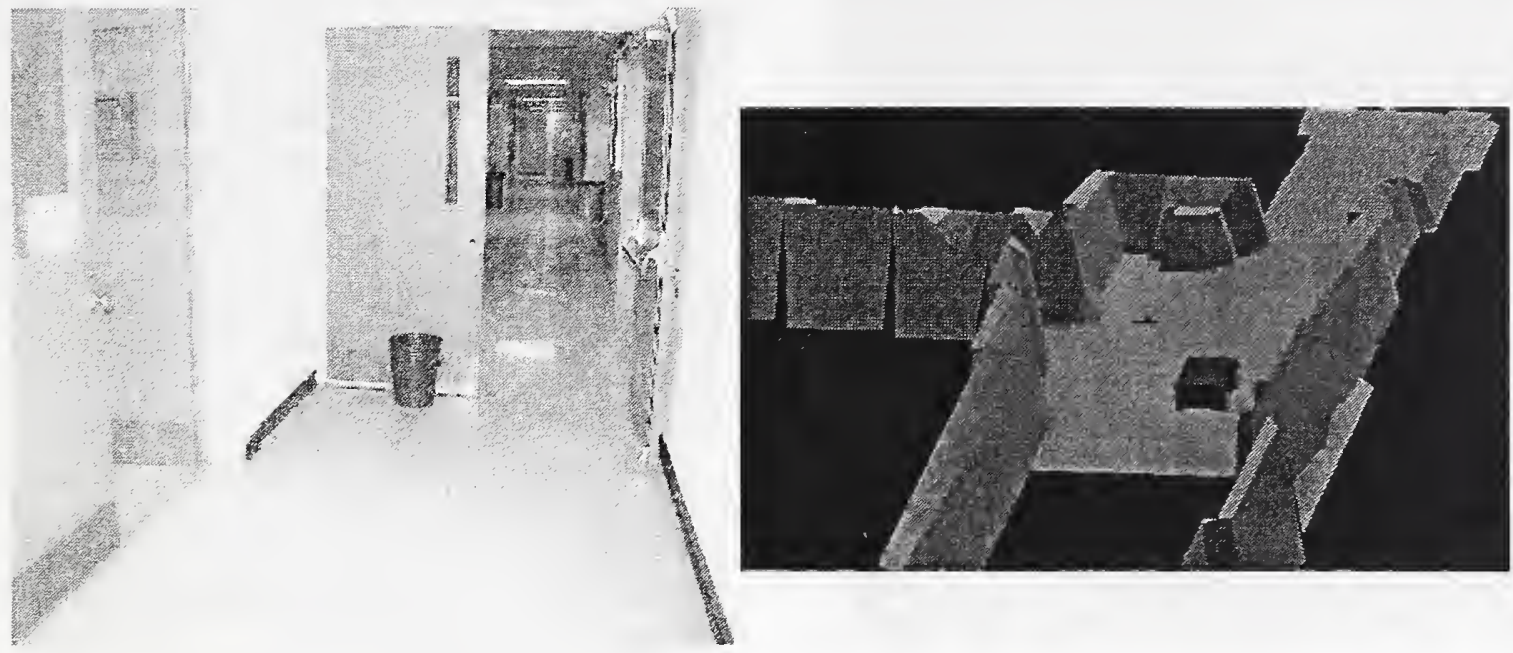

Figure 4. Hallway and 30 map after robot has moved 10 meters

Figure 4 shows the elevation map after the robot has traveled some distance. The photo on the left shows a side doorway leading into a laboratory area; the back wall shows up clearly in the elevation map.

Note that the wastebasket and the doorknob on the left door are sensed and represented in the 3D map. These are objects that might be missed by scanning in a single horizontal plane. The doorknob, for example, looks like a pillar in the $3 \mathrm{D}$ map as it is projected to the floor. This is the correct representation for a cylindrical robot. For a robot that has a varying cross section, a more complex $3 \mathrm{D}$ model could be constructed that would represent overhangs that part or all of the robot could pass under without collision. The important point is that all of the three dimensional features of the world below the height of the sensor that might be obstacles for a robot are captured by this scanning technique and can then be represented in an appropriate way for path planning.

Since the scanning sensor can only see forward and down, it is vulnerable to overhanging obstacles at or above the height of the sensor. Putting the sensor on a tilting platform or adding a second sensor looking up from below or complementary sonar sensors would be needed to detect all possible obstacles that might endanger the robot. Since there are "stealth" objects for any single sensing modality, a multiplicity of sensors is always the best idea in any case.
A final example is shown in Figure 5. This is data collected by moving the sensor, angled downward at $12^{\circ}$, along a bridge between two buildings. The structure of the bridge is a series of pillars that hold windows to the outside and that support the floor to the inside. There is an open gap between the floor and the windows. A handrail runs between the pillars. The scanned data then creates an apparently hollow panel wall, where the inner surface is the data from the handrail projected to the floor. Note that even though the exterior wall is transparent glass, the sensor sees and maps that wall; it also maps external structures (tree branches) seen through the glass. These data were taken in direct sunlight, representing an extremely difficult environment for an optical sensor. 


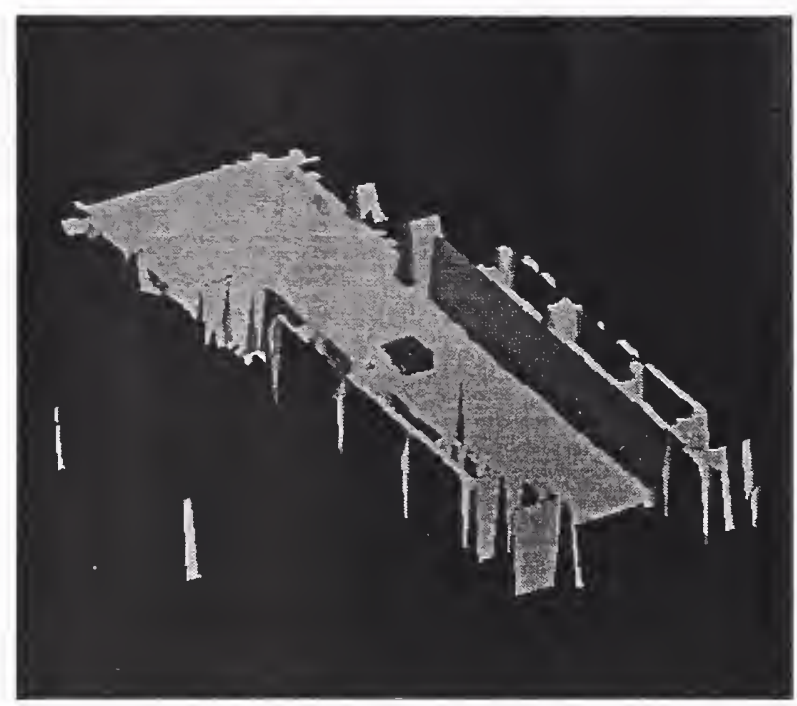

Figure 5. Partial 3D map of bridge with glass walls

\section{Obstacle Avoidance vs Localization and Mapping of Unknown Environments.}

The work described here is targeted at obstacle avoidance, capturing three-dimensional features of an indoor environment in a way that overcomes some of the problems encountered in real world mobile robot applications.

We have not discussed using the data for localization, although it is obvious that the data are very clean and wall following would be straightforward. Similarly, wall features are clearly defined and hence could be used for registration along the length of a hallway. Given an a priori map, created when a robot is installed, the data are adequate for localization and navigation in indoor hallway environments.

We have also not discussed the simultaneous localization and mapping (SLAM) problem, which is mapping of an unknown environment. The issues of dead reckoning accuracy and data registration using Kalman filtering are the same no matter what sensing modality is employed. Scanning laser range data has been used for SLAM in a planar configuration by Konolige [8] and others. Using the scanner in a tilted configuration would be identical once the data are in a $21 / 2 \mathrm{D}$ form in vehicle coordinates.

\section{Conclusion}

A scanning laser range sensor mounted on a moving robot at a downward looking angle can be used to sweep out a volume of space as the robot moves. This will capture the three dimensional structure of the world and identify obstacles that might be a problem for the robot and that would be missed by scanning in a single horizontal plane.

\section{References:}

[1] Nilsson, Nils J., "A Mobile Automaton: An Application of Artificial Intelligence Techniques." Proceedings, IJCAI-1, 1969.

[2] Crowley, James L., "Navigation for an Intelligent Mobile Robot." IEEE Journal of Robots and Automation, 1, 1, p31, 1985.

[3] Moravec, Hans P. and Elfes, Alberto, "High Resolution Maps from Wide Angle Sonar." Proc. 1985 IEEE ICRA, St. Louis, 1985.

[4] Drumheller, M., "Mobile Robot Localization Using Sonar." AI Memo 286, MIT AI Lab, January 1985.

[5] Moravec, H. P., "The Stanford Cart and CMU Rover." Proc. IEEE, 71, 7, p872, July, 1983. Also CMU Tech Report of same title, 1983.

[6] Thorpe, C., et. al., "Vision and Navigation for the Carnegie Mellon NavLab." IEEE PAMI, 10, 3, May, 1988.

[7] Dickmanns, E. D., "A General Dynamic Vision Architecture for UGV and UAV." Journal of Applied Intelligence, 2, p.251, 1992.

[8] Konolige, Kurt, Presentation at DARPA Workshop on Cooperative Robots, February, 2001.

[9] Sick Optic Laser Measurement System LMS 200. Specifications. www.sickoptic.com.

[10] Thrun, Sebastian, "MINERVA: A SecondGeneration Museum Tour-Guide Robot." Proceedings, IEEE ICRA 1999, p.1999.

[11] Thrun, S., Burgard, W. and Fox, D., "A Real

Time Algorithm for Mobile Robot Mapping With Application to Multi-Robot and 3D Mapping." Proceedings, IEEE ICRA 2000, p.321.

[12] Ye, C. and Borenstein, J., 2002, "Characterization of a 2-D Laser Scanner for Mobile Robot Obstacle Negotiation." Proceedings of the 2002 IEEE International Conference on

Robotics and Automation, Washington DC, USA, 11 - 15 May 2002, pp. 2512-2518. 
[13] Albus, J. S., 4-D/RCS: A Reference Model Architecture for Demo III, Proceedings of the ISIC/CIRA/ISAS ' 98 Conference, Gaithersburg, MD, September 14-17, 1998.

[14] Hong, T., Balakirsky, S., Messina, E., Chang, T., and Shneier, M., "A Hierachical World Model for an Autonomous Scout Vehicle," SPIE Aerosense Symposium, Orlando, FL, April, 2002.

[15] Coombs, D., Murphy, K., Lacaze, A., Legowik, S., "Driving Autonomously Off-road Up to $35 \mathrm{~km} / \mathrm{h}$." Proceedings, Intelligent Vehicles Conference, Dearborn, MI, October 4-5, 2000.

[16] Evans, J.M., and Krishnamurthy, B., "Helpmate, the trackless Robotic Courier: A Perspective on the Development of a Commercial Autonomous Mobile Robot." Autonomous Robotic Systems, Ed. Anibal T. de Almeida and Oussama Khatib. p.182 in London, Springer Verlag Lecture Notes in Control and Information Sciences 236, 1998.

[17] Oskard, D., Hong, T., and Shaffer, C., "Realtime Algorithms and Data Structures for Under water Mapping," National Bureau of Standards, 1990. 


